\title{
Freshwater mollusc diversity in the Kruger National Park: \\ a comparison between a period of prolonged drought and a period of exceptionally high rainfall
}

\author{
K.N. De Kock, C.T. Wolmarans and L.H. DU Preez
}

De Kock, K.N., C.T. Wolmarans and L.H. du Preez. 2002. Freshwater mollusc diversity in the Kruger National Park: a comparison between a period of prolonged drought and a period of exceptionally high rainfall. Koedoe 45(2): 1-11. Pretoria. ISSN 0075-6458.

Most of the previous records of the freshwater molluscs from the Kruger National Park date back to 1966 and earlier. On account of several droughts between 1966 and 1995, a survey was done in 1995 to evaluate the effect of these droughts on the mollusc population. As a result of extensive rainfall between 1995 and 2000 another survey was conducted to establish the effect of a period of exceptionally high rainfall on the species' diversity of the mollusc population. With the exception of three habitats, an increase in mollusc diversity was recorded for all the other habitats previously surveyed. One of the invader freshwater snail species, Aplexa marmorata, collected in only one habitat in 1995, was found in seven habitats located mainly in the south during the 2001 survey. Another interesting finding was that juvenile specimens of Lanistes ovum, of which large specimens were present prior to 1966 but none found in 1995, were present in the Sirheni Dam in 2001. From this study the positive effect of the high rainfall on the species' diversity is highly evident.

Key words: Mollusca, freshwater molluscs, invader species, rainfall, Kruger National Park, South Africa.

K.N. de Kock, C.T. Wolmarans and L.H. du Preez, School of Environmental Sciences and Development (Zoology), Potchefstroom University for Christian Higher Education, Private Bag X6001, Potchefstroom 2520, Republic of South Africa.

\section{Introduction}

The importance of regular, normal rainfall to maintain the bio-diversity in conservation areas in South Africa cannot be over-emphasized, especially for those aquatic organisms which lack the ability to migrate to other habitats during periods of serious drought. This inability to migrate leads to the situation where re-population of isolated habitats, such as dams and pans, is largely dependent on time and passive dispersal of biota. Certain freshwater invertebrates, however, have the ability to aestivate, a strategy enabling them to survive prolonged periods of drought. Apart from these freshwater snail species, among others, Bulinus reticulatus Mandahl-Barth, B. forskalii (Ehrenberg, 1831), B. tropicus (Krauss, 1848) and the $B$. africanus group of species (Brown 1994), it is mostly the eggs of some freshwater crustaceans such as Triops, Eocyzicus and Branchipodopsis which can survive periods of drought (Davies \& Day 1997). With regard to the freshwater molluscs, it is known that the largest bio-diversity is to be found in the tropical and sub-tropical areas of the world. In South Africa it is mainly the Limpopo, Mpumalanga and KwaZulu-Natal provinces that represent the tropical and subtropical regions with the Kruger National Park situated in both the first two mentioned provinces. A large variety of freshwater molluscs collected up to 1966 were reported by Oberholzer \& Van Eeden (1967) for the Kruger National Park. 


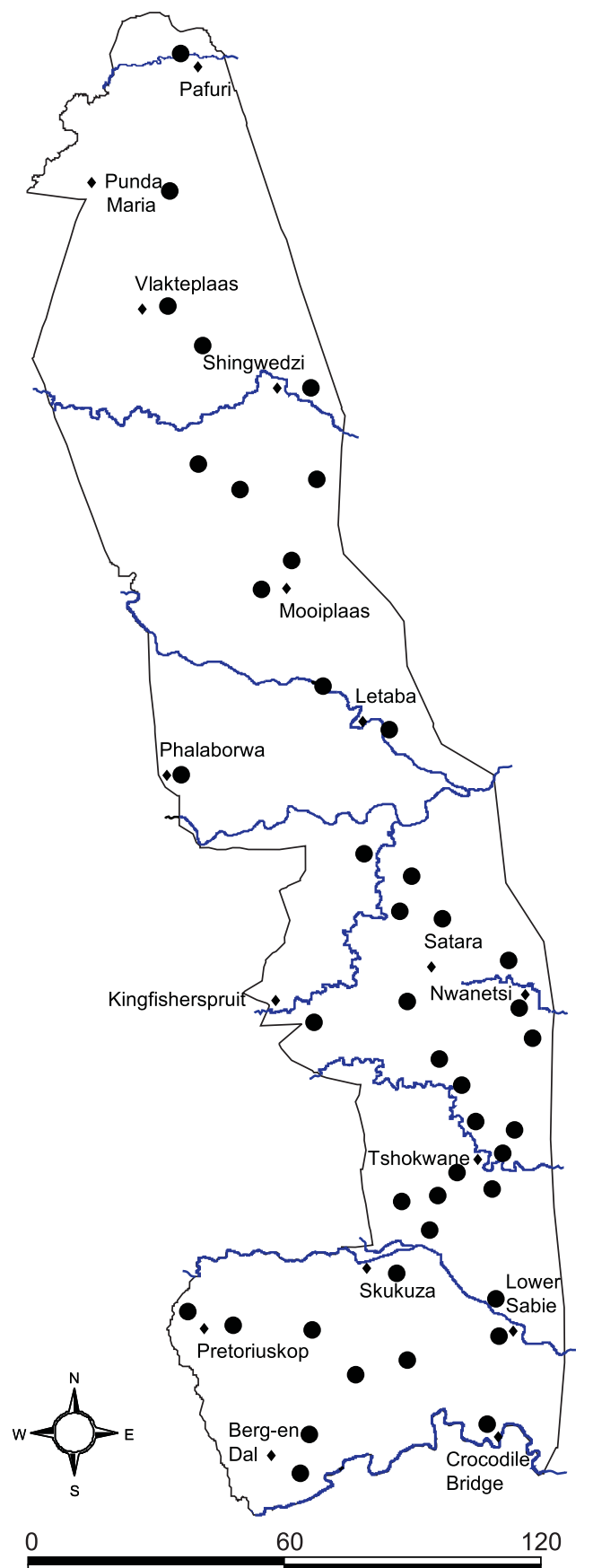

Fig. 1. Water bodies $(\bullet)$ sampled during the 2001 survey. Rainfall monitoring stations $(\bullet)$.
The distribution of freshwater molluses is not a static process and is mainly influenced by the availability of suitable water habitats. The most recent account of the diversity of freshwater molluses in the park, prior to the 2001 survey, dates back to 1995 and is a report by De Kock \& Wolmarans (1998) on a survey done after the drought of 1991-1992. Between 1966 and 1995, the park was struck by several droughts, namely those in 1982-1983 and 1986-1987 - the former also being the severest-prior to the third drought of 1991-1992. According to Zambatis \& Biggs (1995) several of the rainfall monitoring stations recorded the lowest rainfall in history. Between 1995 and 2001, however, average to above normal high rainfall figures were recorded for most of the rainfall monitoring stations. This situation prompted the idea to do a follow-up of the 1995 survey to evaluate the effect of the availability of more permanent water habitats on the freshwater mollusc diversity, and to compare results with the 1995 survey.

\section{Methods and materials}

During the present investigation we concentrated mainly on water bodies that had been surveyed for snails during the 1995 collection. We also concentrated on several other water bodies that were dry during the 1995 survey, or seemed suitable for supporting molluses but of which there were no previous records in the National Freshwater Snail Collection (NFSC). A total of 43 habitats were surveyed during 2001. In each survey, all the habitat types were screened for molluscs by means of aluminium mollusc scoops. The collected molluscs were identified by the senior author who had mainly been responsible for the identification of the molluscs in the NFSC since 1973. The molluscs were subsequently incorporated into the National Freshwater Snail Collection.

Habitats without surface water were screened for mollusc shells. Rainfall records, for the periods 1982-1983, 1985-1987, 1991-1994 and 19952001, were obtained from the Kruger National Park for the monitoring stations nearest to the habitats surveyed. 


\section{Results}

Water bodies sampled during the 2001 survey are depicted in Fig. 1. The results of this investigation are presented in Tables 1, 2 \& 3 and Figs. 2-5. A comparison of the species diversity recorded during the 1995 survey with that of the present survey (Table 1) clearly shows a substantial increase in the diversity in most of the dams and rivers since 1995. A decrease in the species diversity during the 2001 survey is only evident for the Sabie River, the Nwaswitsontso River and the Orpen Dam (which forms part of the Nwaswitsontso River drainage system). During 1995, molluscs were found in only eight of the 24 sites surveyed during both years, while mollucs were present in all of these habitats in 2001. Taking into account the number of habitats sampled during both surveys, it is evident that $B$. forskalii was present in 16 of the 24 habitats surveyed in 2001, while it was recovered from only four of the habitats during the 1995 survey (Table 1). Furthermore, this snail was recorded from nine of the 18 habitats surveyed for the first time in 2001. With regard to its distribution in 1995 , it is clear that it was only found in the southern part of the area, while it was more or less evenly distributed throughout the whole area in 2001 (Fig. 2). In contrast to this, Physa acuta Draparnaud, 1805, which is well known for its invading potential, in spite of exceptionally high mean rainfall for the last five years (Table 4), was present in only two of the habitats, while it was found only once during the 1995 survey (Fig. 3). Aplexa marmorata (Guilding, 1828) which was present only in the Sabie River during the 1995 survey was collected in seven habitats located mainly in the south during the 2001 survey (Fig. 4). Lymnaea columella Say, 1817 another invader species, was recorded only three times before 1966 in the Kruger National Park and only once during the 1995 survey (Fig. 5). In contrast to this, it was collected in eight habitats during the 2001 survey.

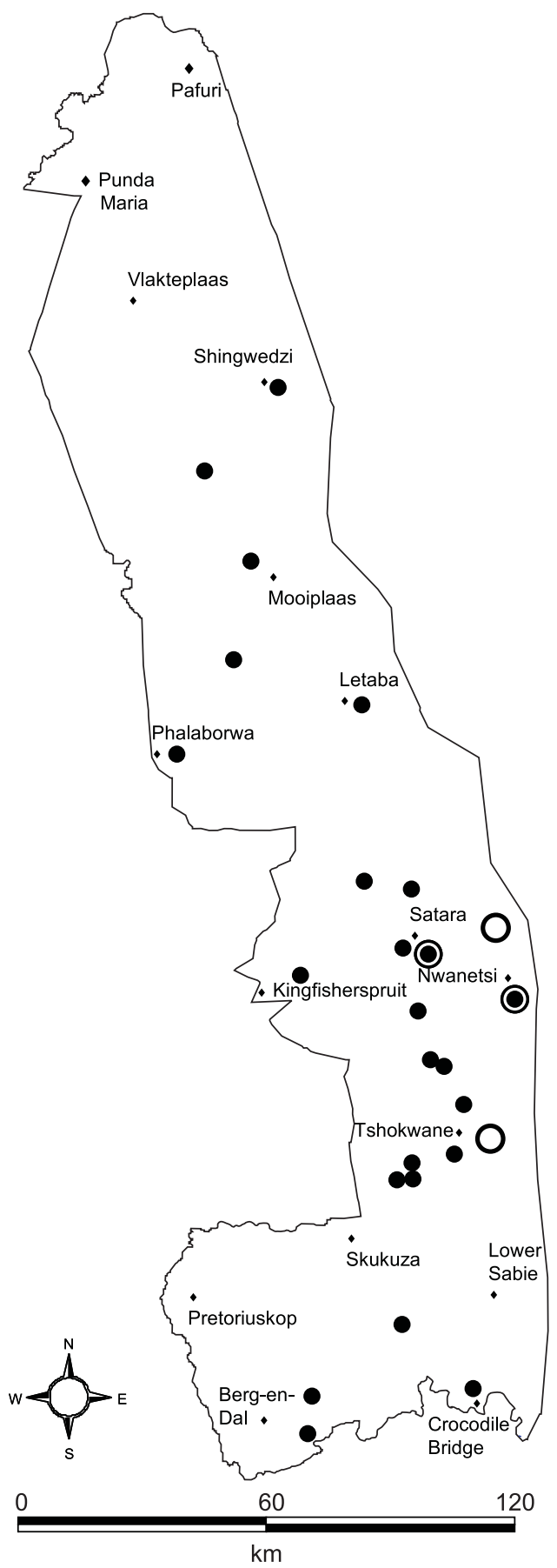

Fig. 2. Distribution of Bulinus forscali in 1995 (O) and $2001(\bullet)$. Rainfall monitoring stations (•). 
Table 1

Habitats of the Kruger National Park surveyed in both 1995 and 2001

\begin{tabular}{|c|c|c|c|}
\hline Rivers & Grid reference & 1995 & 2001 \\
\hline Matjulu Spruit & $25^{\circ} 21^{\prime} 26^{\prime \prime} \mathrm{S} ; 31^{\circ} 31^{\prime} 11^{\prime \prime} \mathrm{E}$ & No molluscs & $\begin{array}{l}\text { Bulinus globosus } \\
\text { Bulinus forskalii } \\
\text { Biomphalaria pfeifferi } \\
\text { Lymnaea natalensis } \\
\text { Lymnaea columella } \\
\text { Aplexa marmorata } \\
\text { Burnupia caffra } \\
\text { Melanoides tuberculata }\end{array}$ \\
\hline Mbyamiti River & $25^{\circ} 13^{\prime} 27^{\prime \prime S} ; 31^{\circ} 34^{\prime} 38^{\prime \prime E}$ & $\begin{array}{l}\text { Bulinus globosus } \\
\text { Spathopsis wahlbergi }\end{array}$ & $\begin{array}{l}\text { Bulinus globosus } \\
\text { Lymnaea columella } \\
\text { Aplexa marmorata }\end{array}$ \\
\hline Mlambane Spruit & $25^{\circ} 21^{\prime} 26^{\prime \prime S} ; 31^{\circ} 31^{\prime} 11^{\prime \prime E}$ & No molluscs & $\begin{array}{l}\text { Bulinus forskalii } \\
\text { Biomphalaria pfeifferi } \\
\text { Aplexa marmorata }\end{array}$ \\
\hline Nwanetsi River & $24^{\circ} 25^{\prime \prime} 12^{\prime \prime S} ; 31^{\circ} 47^{\prime} 01^{\prime \prime E}$ & $\begin{array}{l}\text { Bulinus globosus } \\
\text { Bulinus natalensis } \\
\text { Bulinus forskalii } \\
\text { Biomphalaria pfeifferi } \\
\text { Lymnaea natalensis } \\
\text { Burnupia sp } \\
\text { Melanoides tuberculata } \\
\text { Cleopatra bulimoides } \\
\text { Spathopsis wahlbergi } \\
\text { Spathopsis petersi } \\
\text { Corbicula fluminalis } \\
\text { Eupera ferruginea }\end{array}$ & $\begin{array}{l}\text { Bulinus tropicus } \\
\text { Bulinus forskalii } \\
\text { Biomphalaria pfeifferi } \\
\text { Ceratophallus natalensis } \\
\text { Burnupia caffra } \\
\text { Melanoides tuberculata } \\
\text { Spathopsis petersi } \\
\text { Corbicula fluminalis } \\
\text { Oxyloma striata }\end{array}$ \\
\hline Nwaswitsontso River & $24^{\circ} 37^{\prime} 10^{\prime \prime S} ; 31^{\circ} 47^{\prime} 28^{\prime \prime E}$ & $\begin{array}{l}\text { Cleopatra ferruginea } \\
\text { Spathopsis petersi }\end{array}$ & Bulinus forskalii \\
\hline Sabie River & $25^{\circ} 07^{\prime} 06^{\prime \prime S} ; 31^{\circ} 55^{\prime} 00^{\prime \prime} \mathrm{E}$ & $\begin{array}{l}\text { Bulinus globosus } \\
\text { Biomphalaria pfeifferi } \\
\text { Lymnaea natalensis } \\
\text { Lymnaea columella } \\
\text { Physa acuta } \\
\text { Aplexa marmorata } \\
\text { Melanoides tuberculata } \\
\text { Unio caffer } \\
\text { Eupera ferruginea } \\
\text { Oxyloma patentissima }\end{array}$ & $\begin{array}{l}\text { Physa acuta } \\
\text { Aplexa marmorata }\end{array}$ \\
\hline Timbavati River & $24^{\circ} 13^{\prime} 50^{\prime \prime} \mathrm{S} ; 31^{\circ} 38^{\prime} 03^{\prime \prime E}$ & No molluscs & $\begin{array}{l}\text { Bulinus africanus } \\
\text { Lymnaea natalensis } \\
\text { Burnupia caffra }\end{array}$ \\
\hline Engelhard Dam & $23^{\circ} 50^{\prime} 28^{\prime \prime} \mathrm{S} ; 31^{\circ} 38^{\prime} 07^{\prime \prime} \mathrm{E}$ & No molluscs & $\begin{array}{l}\text { Bulinus globosus } \\
\text { Bulinus tropicus } \\
\text { Bulinus forskalii } \\
\text { Biomphalaria pfeifferi } \\
\text { Physa acuta } \\
\text { Melanoides tuberculata } \\
\text { Corbicula fluminalis } \\
\text { Pisidium pirothi } \\
\text { Bulinus tropicus }\end{array}$ \\
\hline
\end{tabular}




\begin{tabular}{|c|c|c|c|}
\hline Rivers & Grid reference & 1995 & 2001 \\
\hline Gezantombi Dam & $25^{\circ} 20^{\prime} 05^{\prime \prime} \mathrm{S} ; 31^{\circ} 52^{\prime} 35^{\prime \prime} \mathrm{E}$ & No molluscs & $\begin{array}{l}\text { Bulinus forskalii } \\
\text { Lymnaea. columella } \\
\text { Aplexa marmorata } \\
\text { Burnupia caffra }\end{array}$ \\
\hline Gudzani Dam & $24^{\circ} 22^{\prime} 05^{\prime \prime S} ; 31^{\circ} 55^{\prime} 33^{\prime \prime} \mathrm{E}$ & $\begin{array}{l}\text { Bulinus globosus } \\
\text { Bulinus forskalii } \\
\text { Biomphalaria pfeifferi } \\
\text { Lymnaea natalensis } \\
\text { Spathopsis petersi } \\
\text { Unio caffer }\end{array}$ & $\begin{array}{l}\text { Bulinus globosus } \\
\text { Bulinus tropicus } \\
\text { Biomphalaria pfeifferi } \\
\text { Lymnaea natalensis } \\
\text { Ceratophallus natalensis } \\
\text { Melanoides tuberculata } \\
\text { Spathopsis petersi } \\
\text { Corbicula fluminalis }\end{array}$ \\
\hline Klopperfontein Dam & $22^{\circ} 37^{\prime} 42^{\prime \prime} \mathrm{S} ; 31^{\circ} 10^{\prime} 20^{\prime \prime} \mathrm{E}$ & No molluscs & $\begin{array}{l}\text { Bulinus tropicus } \\
\text { Bulinus forskalii }\end{array}$ \\
\hline Krapkuil Dam & $23^{\circ} 14^{\prime} 43^{\prime \prime} \mathrm{S} ; 31^{\circ} 18^{\prime} 07^{\prime \prime} \mathrm{E}$ & No molluscs & Burnupia caffra \\
\hline Kumana Dam & $24^{\circ} 37^{\prime} 11^{\prime \prime S} ; 31^{\circ} 47^{\prime} 40^{\prime \prime} \mathrm{E}$ & No molluscs & Bulinus forskalii \\
\hline Manzimahle Dam & $24^{\circ} 53^{\prime} 51^{\prime \prime S} ; 31^{\circ} 43^{\prime} 55^{\prime \prime} \mathrm{E}$ & No molluscs & Bulinus forskalii \\
\hline Mazithi Dam & $24^{\circ} 43^{\prime} 20^{\prime \prime} \mathrm{S} ; 31^{\circ} 50^{\prime} 34^{\prime \prime} \mathrm{E}$ & No molluscs & Bulinus forskalii \\
\hline Nkaya Pan & $24^{\circ} 31^{\prime} 54^{\prime \prime S} ; 31^{\circ} 45^{\prime} 25^{\prime \prime} \mathrm{E}$ & No molluscs & Bulinus forskalii \\
\hline Nsemani Dam & $24^{\circ} 23^{\prime} 32^{\prime \prime} \mathrm{S} ; 31^{\circ} 43^{\prime} 05^{\prime \prime} \mathrm{E}$ & No molluscs & $\begin{array}{l}\text { Bulinus forskalii } \\
\text { Burnupia caffra }\end{array}$ \\
\hline Nwanetsi water hole & $24^{\circ} 28^{\prime} 22^{\prime \prime S} ; 31^{\circ} 58^{\prime} 51^{\prime \prime} \mathrm{E}$ & $\begin{array}{l}\text { Bulinus globosus } \\
\text { Bulinus natalensis } \\
\text { Bulinus forskalii } \\
\text { Biomphalaria pfeifferi } \\
\text { Lymnaea natalensis } \\
\text { Burnupia sp. } \\
\text { Melanoides tuberculata } \\
\text { Cleopatra bulimoides } \\
\text { Spathopsis wahlbergi } \\
\text { Spathopsis petersi } \\
\text { Corbicula fluminalis } \\
\text { Eupera ferruginea }\end{array}$ & $\begin{array}{l}\text { Bulinus globosus } \\
\text { Bulinus tropicus } \\
\text { Bulinus forskalii } \\
\text { Biomphalaria pfeifferi } \\
\text { Ceratophallus natalensis } \\
\text { Burnupia caffra } \\
\text { Spathopsis wahlbergi } \\
\text { Spathopsis petersi } \\
\text { Corbicula fluminalis }\end{array}$ \\
\hline Olifantdrinkgat & $24^{\circ} 53^{\prime} 56^{\prime \prime S} ; 31^{\circ} 44^{\prime} 54^{\prime \prime} \mathrm{E}$ & No molluscs & Bulinus forskalii \\
\hline Orpen Dam & $24^{\circ} 47^{\prime} 31^{\prime \prime S} ; 31^{\circ} 53^{\prime} 51^{\prime \prime E}$ & $\begin{array}{l}\text { Bulinus globosus } \\
\text { Bulinus tropicus } \\
\text { Bulinus forskalii } \\
\text { Biomphalaria pfeifferi } \\
\text { Lymnaea natalensis } \\
\text { Ceratophallus natalensis } \\
\text { Melanoides tuberculata } \\
\text { Spathopsis wahlbergi } \\
\text { Spathopsis petersi }\end{array}$ & $\begin{array}{l}\text { Lymnaea columella } \\
\text { Ceratophallus natalensis }\end{array}$ \\
\hline Pioniers Dam & $23^{\circ} 31^{\prime} 29^{\prime \prime} \mathrm{S} ; 31^{\circ} 23^{\prime} 56^{\prime \prime} \mathrm{E}$ & $\begin{array}{l}\text { Bulinus globosus } \\
\text { Biomphalaria pfeifferi } \\
\text { Lymnaea natalensis }\end{array}$ & $\begin{array}{l}\text { Bulinus globosus } \\
\text { Bulinus forskalii } \\
\text { Biomphalaria pfeifferi }\end{array}$ \\
\hline
\end{tabular}




\begin{tabular}{llll}
\hline Rivers & Grid reference & 1995 & 2001 \\
\hline & & $\begin{array}{l}\text { Melanoides tuberculata } \\
\text { Spathopsis petersi } \\
\text { Corbicula fluminalis }\end{array}$ & $\begin{array}{l}\text { Lymnaea natalensis } \\
\text { Melanoides tuberculata }\end{array}$ \\
Shitlhave Dam & $25^{\circ} 08^{\prime} 38^{\prime \prime} \mathrm{S} ; 31^{\circ} 19^{\prime} 31^{\prime \prime} \mathrm{E}$ & No molluscs & $\begin{array}{l}\text { Biomphalaria pfeifferi } \\
\text { (shell) }\end{array}$ \\
Silolweni Dam & $24^{\circ} 29^{\prime} 21^{\prime \prime} \mathrm{S} ; 31^{\circ} 50^{\prime} 11^{\prime \prime} \mathrm{E}$ & No molluscs & $\begin{array}{l}\text { Bulinus forskalii } \\
\text { Burnupia caffra }\end{array}$ \\
Vervoer Dam & $25^{\circ} 07^{\prime} 07^{\prime \prime S} ; 31^{\circ} 29^{\prime} 29^{\prime \prime} \mathrm{E}$ & No molluscs & $\begin{array}{l}\text { Lymnaea columella } \\
\text { Aplexa marmorata }\end{array}$ \\
\hline
\end{tabular}

\section{Discussion}

From the results of this investigation it is clear that the high rainfall average recorded for 1995-2001 period had a significant influence on the number of habitats from which molluscs were recorded as compared to the 1995 survey. For the majority of the habitats the mean rainfall for the 2001 period was nearly double the figures recorded for the 1995 period. The decrease in snail bio-diversity in the Sabie and Nwaswitsiontso rivers and Orpen Dam (which forms part of the Nwaswitsontso River drainage system, as previously mentioned) could probably be ascribed to heavy floods in these habitats prior to the present survey.

In the same localities investigated, $B$. forskalii was found only four times in 1995 as compared to 16 times in 2001 and was, furthermore, present in nine of the 18 habitats surveyed for the first time in 2001. This snail is able to exploit a wide variety of natural and artificial habitats, including the margins of lakes and permanent swamps, but is most abundant in small water bodies and was also collected in alluvial pools associated with rice cultivation in Gambia (Brown 1994). The fact that this snail was found in many more habitats during the 2001 survey may be ascribed to its ability to aestivate through long periods of drought. However, the possibility that some of these habitats were populated by this snail species for the first time due to the high rainfall of the 19952001 period cannot be ruled out.

Aplexa marmorata, a confirmed invader species collected only once during the 1995 survey, was present in seven of the localities in 2001, which was investigated during both surveys. Although it was collected several times during the past 15 years in KwaZuluNatal, it was recorded only once from the Limpopo Province (Dana 1999) at the time of the present investigation. The fact that this species was found in a number of habitats during the 2001 survey seems to suggest that it could be in the process of becoming well established in the southern parts of the Kruger National Park. Whether it has already invaded habitats in the adjacent Swaziland needs further investigation. Although this species is usually reported from water bodies modified by man (Dana 1999), it was present in several rivers and dams in the Kruger National Park

In contrast to this, P. acuta, which has a worldwide distribution and which is established in many African rivers and lakes from South Africa to Morocco, and is considered the second most successful invader snail species in South Africa (De Kock et al. 1989), was recovered from only two habitats during the 2001 survey as compared to the one in 1995. Its distribution pattern in the 
Table 2

Molluscs found in waterbodies of the Kruger National Park surveyed for the first time in 2001

\begin{tabular}{|c|c|c|}
\hline Waterbodies & Grid reference & 2001 \\
\hline Grootvlei Dam & $23^{\circ} 20^{\prime} 41^{\prime \prime S} ; 31^{\circ} 30^{\prime} 56^{\prime \prime E}$ & Spathopsis sp. \\
\hline Jones se Dam & $24^{\circ} 51^{\prime} 23^{\prime \prime S} ; 31^{\circ} 45^{\prime} 13^{\prime \prime E}$ & $\begin{array}{l}\text { Bulinus forskalii } \\
\text { Ceratophallus natalensis } \\
\text { Burnupia caffra }\end{array}$ \\
\hline Kanniedood Dam & $23^{\circ} 08^{\prime} 40^{\prime \prime S} ; 31^{\circ} 27^{\prime} 48^{\prime \prime} \mathrm{E}$ & $\begin{array}{l}\text { Bulinus tropicus } \\
\text { Bulinus forskalii } \\
\text { Lymnaea natalensis }\end{array}$ \\
\hline Letaba River & $23^{\circ} 45^{\prime} 28^{\prime \prime S} ; 31^{\circ} 22^{\prime} 20^{\prime \prime E}$ & $\begin{array}{l}\text { Bulinus forskalii } \\
\text { Physa acuta } \\
\text { Melanoides tuberculata }\end{array}$ \\
\hline Mestel Dam & $25^{\circ} 06^{\prime} 54^{\prime \prime} \mathrm{S} ; 31^{\circ} 12^{\prime} 50^{\prime \prime} \mathrm{E}$ & $\begin{array}{l}\text { Bulinus globosus } \\
\text { Lymnaea columella } \\
\text { Aplexa marmorata }\end{array}$ \\
\hline Mpondo Dam & $25^{\circ} 12^{\prime} 12^{\prime \prime S} ; 31^{\circ} 43^{\prime} 08^{\prime \prime E}$ & $\begin{array}{l}\text { Bulinus forskalii } \\
\text { Lymnaea natalensis } \\
\text { Lymnaea columella } \\
\text { Spathopsis petersi }\end{array}$ \\
\hline Mtombeni Spruit & $24^{\circ} 15^{\prime} 40^{\prime \prime S} ; 31^{\circ} 43^{\prime} 40^{\prime \prime E}$ & Bulinus forskalii \\
\hline Nshawu Dam & $23^{\circ} 31^{\prime} 40^{\prime \prime} \mathrm{S} ; 31^{\circ} 29^{\prime} 09^{\prime \prime} \mathrm{E}$ & Ceratophallus natalensis \\
\hline Olifantsdrinkbad & $23^{\circ} 19^{\prime} 45^{\prime \prime S} ; 31^{\circ} 19^{\prime} 44^{\prime \prime E}$ & Bulinus forskalii \\
\hline Piet Grobler Dam & $24^{\circ} 13^{\prime} 54^{\prime \prime S} ; 31^{\circ} 38^{\prime} 03^{\prime \prime E}$ & Bulinus forskalii \\
\hline Rabelais Dam & $24^{\circ} 27^{\prime} 30^{\prime \prime} \mathrm{S} ; 31^{\circ} 29^{\prime} 58^{\prime \prime} \mathrm{E}$ & $\begin{array}{l}\text { Bulinus tropicus } \\
\text { Bulinus forskalii }\end{array}$ \\
\hline Sable Dam & $23^{\circ} 56^{\prime} 12^{\prime \prime S} ; 31^{\circ} 14^{\prime} 04^{\prime \prime E}$ & $\begin{array}{l}\text { Bulinus tropicus } \\
\text { Bulinus forskalii }\end{array}$ \\
\hline Shisa Spruit & $22^{\circ} 57^{\prime} 32^{\prime \prime S} ; 31^{\circ} 15^{\prime} 01^{\prime \prime E}$ & $\begin{array}{l}\text { Cleopatra ferruginea } \\
\text { Melanoides tuberculata } \\
\text { Spathopsis petersi } \\
\text { Corbicula fluminalis }\end{array}$ \\
\hline Sirheni Dam & $22^{\circ} 56^{\prime} 57^{\prime \prime S} ; 31^{\circ} 14^{\prime} 02^{\prime \prime E}$ & $\begin{array}{l}\text { Bulinus globosus } \\
\text { Biomphalaria pfeifferi } \\
\text { Lymnaea natalensis } \\
\text { Lymnaea columella } \\
\text { Lanistes ovum }\end{array}$ \\
\hline Sunset Dam & $25^{\circ} 06^{\prime} 57^{\prime \prime S} ; 31^{\circ} 54^{\prime} 41^{\prime \prime E}$ & Aplexa marmorata \\
\hline
\end{tabular}

Table 3

Waterbodies of the Kruger National Park surveyed for the first time in 2001 and in which no molluscs were found

\begin{tabular}{ll}
\hline Waterbodies & Grid reference \\
\hline Luvuvhu River & $22^{\circ} 26^{\prime} 38^{\prime \prime} \mathrm{S} ; 31^{\circ} 13^{\prime} 02^{\prime \prime} \mathrm{E}$ \\
Ngotso Dam & $24^{\circ} 10^{\prime} 31^{\prime \prime} \mathrm{S} ; 31^{\circ} 43^{\prime} 09^{\prime \prime} \mathrm{E}$ \\
Nhlanganzwane Dam & $25^{\circ} 14^{\prime} 40^{\prime \prime} \mathrm{S} ; 31^{\circ} 58^{\prime} 30^{\prime \prime} \mathrm{E}$ \\
\hline
\end{tabular}




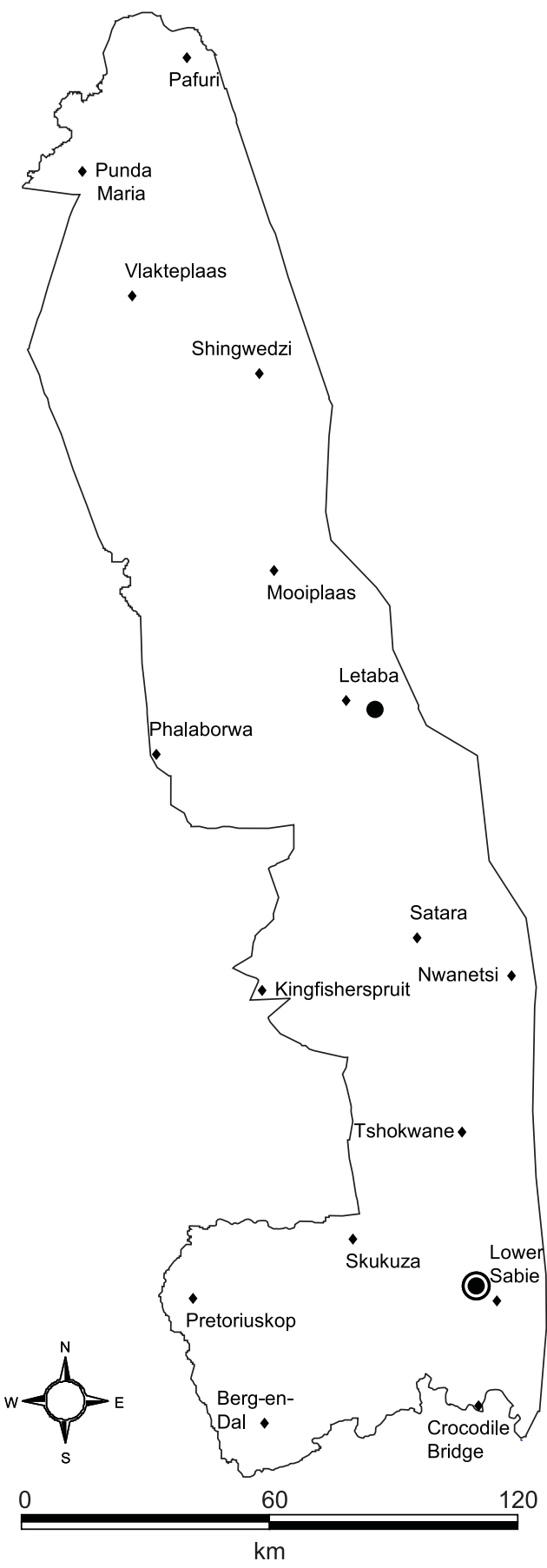

Fig. 3. Distribution of Physa acuta in 1995 (O) and $2001(\bullet)$. Rainfall monitoring stations $(\bullet)$.
RSA shows concentrations of collections around major urban areas (Brackenbury \& Appleton 1993). It is now found in various artificial and natural habitat types and taking into account that this species is not highly specific with regard to its habitat preferences it is quite surprising that it has a relatively limited distribution in the Kruger National Park. However, it occurs commonly in polluted water and has therefore been nicknamed the sewage snail and seems to have difficulties in establishing itself far from human works or habitats (Brown 1978; Brackenbury \& Appleton 1993). This could be one of the reasons for its limited occurrence in the Kruger National Park, especially with regard to the dams and rivers, which originate in or near the park. Although the drought of 1995 may also serve as an explanation for this situation, it must be kept in mind that it had been recovered only three times from the Kruger National Park prior to 1966.

Lymnaea columella, which is considered the most successful invader snail species in South Africa (Brown 1967) was recovered from five habitats during the 2001 survey and from only one during the 1995 survey. Scrutiny of its distribution in the whole of the RSA (De Kock et al. 1989) shows clearly why it has earned the title as the most successful invader snail species, because it has successfully established itself in vast areas of the country since its introduction in the early 1940s. Before 1966 it was found in 132 loci, and in 225 additional loci from 1966 up to the present (De Kock et al. 1989). This species was first reported from the Kruger National Park in 1967 from three localities by Oberholzer \& Van Eeden (1967) and was collected in only three additional localities during the 2001 survey. In contrast to this, $A$. marmorata was collected in six additional localities since the 1995 survey. This increase in collection sites over a period of only six years seems to suggest that $A$. marmorata could become just as successful as $L$. columella in establishing itself in this country. 


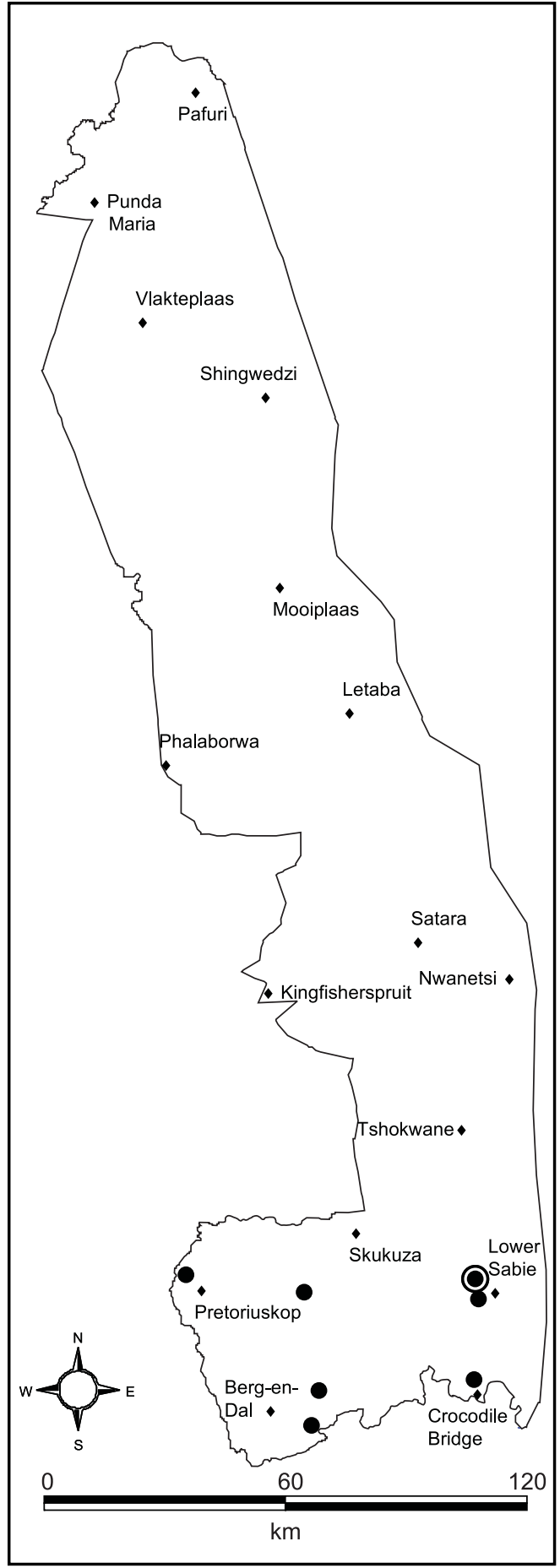

Fig. 4. Distribution of Aplexa marmorata in 1995 $(\mathrm{O})$ and $2001(\bullet)$. Rainfall monitoring stations (•).

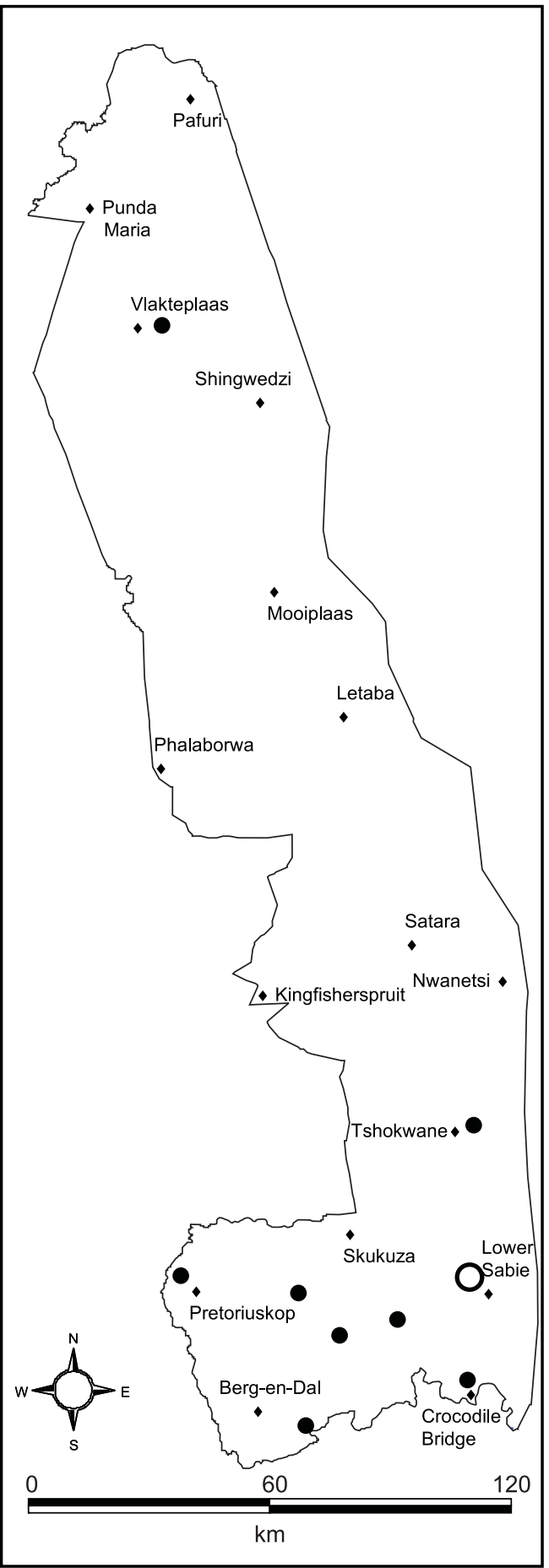

Fig. 5. Distribution of Lymnea columella in 1995 (O) and $2001(\bullet)$. Rainfall monitoring stations (•). 
Table 4

Rainfall records of the Kruger National Park at the different monitoring stations nearest to collection sites over different time periods

\begin{tabular}{|c|c|c|c|c|c|c|}
\hline $\begin{array}{l}\text { Rainfall } \\
\text { monitoring } \\
\text { station }\end{array}$ & Collection site & $\begin{array}{l}\text { Average rain- } \\
\text { fall of all } \\
\text { records avail- } \\
\text { able }(\mathrm{mm})\end{array}$ & $\begin{array}{c}\text { Average } \\
\text { rainfall for } \\
1882-1983 \\
(\mathrm{~mm})\end{array}$ & $\begin{array}{c}\text { Average } \\
\text { rainfall for } \\
1985-1987 \\
(\mathrm{~mm})\end{array}$ & $\begin{array}{c}\text { Average } \\
\text { rainfall for } \\
1991-1994 \\
\quad(\mathrm{~mm})\end{array}$ & $\begin{array}{c}\text { Average } \\
\text { rainfall for } \\
1995-2001 \\
(\mathrm{~mm})\end{array}$ \\
\hline Berg-en-Dal & $\begin{array}{l}\text { Matjulu Spruit } \\
\text { Mlambane Spruit } \\
\text { Mpanamana Dam }\end{array}$ & $\mathrm{a}$ & $\mathrm{a}$ & $\mathrm{a}$ & $\mathrm{a}$ & 832 \\
\hline Crocodile Bridge & $\begin{array}{l}\text { Gezantombi Dam } \\
\text { Nhlanganzwanedam }\end{array}$ & 547 & 380 & 521 & 506 & 814 \\
\hline Houtboschrand & $\begin{array}{l}\text { Mtombeni Spruit } \\
\text { Ngotso Dam } \\
\text { Timbavati River }\end{array}$ & 386 & 304 & 355 & 279 & 560 \\
\hline King Fisher Spruit & Rabelais Dam & 523 & 265 & 545 & 440 & 673 \\
\hline Letaba & $\begin{array}{l}\text { Engelhard Dam } \\
\text { Letaba River } \\
\text { Olifants River }\end{array}$ & 435 & 228 & 335 & 281 & 418 \\
\hline Lower Sabie & $\begin{array}{l}\text { Lower Sabie Rest } \\
\text { Camp } \\
\text { Mpondo Dam } \\
\text { Sabie River }\end{array}$ & 577 & 493 & 536 & 441 & 787 \\
\hline Mooiplaas & $\begin{array}{l}\text { Grootvlei Dam } \\
\text { Nshawu Dam } \\
\text { Olifantdrinkbad } \\
\text { Pioniers Dam } \\
\text { Tsende Drif }\end{array}$ & 449 & 196 & 447 & 281 & 663 \\
\hline Nwanetsi & $\begin{array}{l}\text { Gudzani Dam } \\
\text { Nwanetsi River } \\
\text { Nwanetsi water hole }\end{array}$ & 515 & 308 & 437 & 376 & 653 \\
\hline Pafuri & Luvuvhu River & 332 & 98 & 422 & 297 & 652 \\
\hline Phalaborwa & Sable Dam & 523 & 234 & 484 & 412 & 673 \\
\hline Pretoriuskop & $\begin{array}{l}\text { Mbyamiti River } \\
\text { Mestel Dam } \\
\text { Shitlhave Dam }\end{array}$ & 709 & 417 & 428 & 507 & 989 \\
\hline Punda Maria & Klopperfontein Dam & 528 & 142 & 520 & 327 & 722 \\
\hline Satara & $\begin{array}{l}\text { Nkaya Pan } \\
\text { Nsemani Dam } \\
\text { Piet Grobler Dam }\end{array}$ & 526 & 326 & 428 & 507 & 595 \\
\hline Shingwedzi & $\begin{array}{l}\text { Kanniedood Dam } \\
\text { Krapkuil Dam } \\
\text { Sirheni Dam } \\
\text { Sisha Spruit }\end{array}$ & 462 & 248 & 356 & 334 & 704 \\
\hline Skukuza & $\begin{array}{l}\text { Manzimahle Dam } \\
\text { Olifantdrinkgat } \\
\text { Skukuza fish pond } \\
\text { Sunset Dam } \\
\text { Vervoer Dam }\end{array}$ & 533 & 272 & 547 & 411 & 674 \\
\hline Tshokwane & $\begin{array}{l}\text { Jones se Dam } \\
\text { Kumana Dam } \\
\text { Leeupan } \\
\text { Mazithi Dam } \\
\text { Nwaswitsontso River } \\
\text { Orpen Dam } \\
\text { Silolweni Dam }\end{array}$ & 533 & 295 & 547 & 411 & 761 \\
\hline
\end{tabular}


With regard to Lanistes ovum Peters in Troschel, 1845 it needs to be mentioned that large specimens were collected before 1966, while none were found during the 1995 survey. However, juvenile specimens were found in the Sirheni Dam during the 2001 survey which might indicate that this species could be in the process of recovering from the previous droughts. More or less the same conclusions could be drawn for the bivalves on record for the park in general and the Spathopsis spp. in particular.

\section{Conclusions}

From the results of this investigation it is clear that there was a significant increase in the number of localities which yielded molluscs during the present study as compared to the 1995 survey. This could mainly be ascribed to the abnormal high rainfall during 1995-2001 period. With regard to the invader species $A$. marmorata, a follow-up survey in about five years' time is recommended to establish whether the northern parts of the Kruger National Park are also going to be invaded by this species.

\section{Acknowledgements}

The authors wish to thank the following institution and persons for assistance: South African National Parks for permission to conduct the research; Dr Andrew Deacon for coordinating this project on behalf of the Kruger National Park and Mr Nick Zambatis for kindly making the necessary rainfall data available.

\section{References}

Brackenbury, T.D. \& C.C. Appleton. 1993. Recolonization of the Umsindusi River, Natal, South Africa, by the invasive gastropod, Physa acuta (Basommatophora, Physidae). Journal of Medical \& Applied Malacology 5:39-44.

Brown, D.S. 1978. 35. Freshwater molluscs. Pp. 1155-1180. In: WERGER, M.J.A. (Ed.). Biogeography and ecology of southern Africa 1. The Hague: Junk.

Brown, D.S. 1994. Freshwater molluscs of Africa and their medical importance. London: Taylor \& Francis.

DANA, P. 1999. Aplexa marmorata (Guilding, 1828) (Basommatophora: Physidae) - an invasive freshwater snail in South Africa. M.Sc thesis, University of Natal, Durban.

Davies, B. \& J. Day. 1989. Vanishing waters. Cape Town: University of Cape Town Press.

De Kock, K.N., P.H. Joubert. \& S.J. Pretorius. 1989. Geographical distribution and habitat preferences of the invader freshwater snail species Lymnaea columella (Mollusca: Gastropoda) in South Africa. Onderstepoort Journal of Veterinary Research 56: 271-275.

De Kock, K.N. \& C.T. Wolmarans. 1989. A reevaluation of the occurrence of freshwater molluscs in the Kruger National Park. Koedoe 41:1-8.

OBerholzer, G. \& J.A. van EedEN. 1967. The freshwater molluscs of the Kruger National Park. Koedoe 10: 1-42.

Zambatis, N. \& H.C. BigGs. Rainfall and temperatures during the 1991/92 drought in the Kruger National Park. Koedoe 38: 1-16. 